\title{
Influência do Intervalo de Tempo Entre as Sessões de Alongamento no Ganho de Flexibilidade dos Isquiotibiais
}

\author{
Influence of the Time Interval Between Stretching Sessions on \\ Increased Hamstring Flexibility
}

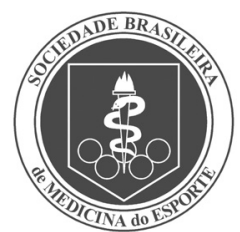

Artigo Original
Zenewton André da Silva Gama', Alexandre Vinícyus Ribeiro Dantas¹, Túlio Oliveira de Souza

1. Universidade Federal do Rio Grande do Norte (UFRN) - Natal / Rio Grande do Norte.

\section{Endereço para correspondência:} Zenewton André da Silva Gama. Departamento de Fisioterapia, Facultad de Medicina. Universidad de Murcia . 30100 Espinardo, Murcia - España.

E-mail: zenewton.andre@alu.um.es

Submetido em 23/08/2007 Versão final recebida em 30/04/2008 Aceito em 24/10/2008

\begin{abstract}
RESUMO
Contexto: A otimização do treino de flexibilidade está relacionada com o uso de parâmetros adequados de alongamento. Entretanto, o intervalo de tempo adequado entre as sessões de alongamento tem sido pouco investigado. Objetivo: Verificar se a variação no intervalo de tempo entre sessões de alongamento influencia no ganho de flexibilidade. Métodos: 28 mulheres, com idade de 22,5 \pm 1,8 anos, foram distribuídas aleatoriamente em três grupos. Aplicaram-se 10 sessões de alongamento nos isquiotibiais do membro direito. $O$ grupo OX $(n=8)$ foi o controle e não recebeu alongamento. O grupo $3 X(n=10)$ alongou três vezes por semana (intervalo de 48 horas) e o grupo 5X ( $n=10$ ), cinco vezes (intervalo de 24 horas). Aplicaram-se 10 sessões de alongamento (sustentar-relaxar) nos isquiotibiais direitos. As medidas foram tomadas por análise fotométrica no programa AutoCad ${ }^{\circledR} 2000$. A análise estatística foi realizada com ANOVA e teste post hoc de Newman-Keuls adotando um p-valor referencial de 0,05. Resultados: Após 10 sessões, identificou-se aumento da flexibilidade nos grupos experimentais, porém sem diferença entre estes. O grupo $3 X$ aumentou significativamente a partir do $10^{\circ}$ dia do programa (quinta sessão) e o grupo $5 X$, a partir do terceiro (terceira sessão). Conclusões: 0 alongamento aumenta a flexibilidade dos isquiotibiais, independente do tempo entre as sessões (24 ou 48 horas); e a variável tempo não influencia o ganho de flexibilidade total. Porém, com cinco sessões semanais, ganha-se flexibilidade mais rapidamente. Isso sugere que o ganho de flexibilidade é sessão-dependente.
\end{abstract}

Palavras-chave: facilitação neuromuscular proprioceptiva, flexibilidade, alongamento.

\begin{abstract}
Context:The optimization of flexibility training is related to the use of ideal stretching parameters. However, the time interval between sessions has been little investigated. Objective: To verify if the variation in time interval between stretching sessions influences in flexibility gain. Methodology: Twenty-eight women, average age of $22.5 \pm 1.8$ years, were randomly distributed into three groups. Group OX was the control and performed no stretching. Group $3 X(n=10)$ took part in three sessions per week (interval $=48$ hours) and group $5 X(n=10)$ in five times (interval $=24$ hours). Ten stretching sessions were applied (hold-relax) to the right hamstring. The measurement of active knee extension was performed using photometric analyses on AutoCAD 2000 software. Statistical analysis was carried out with ANOVA and Newman-Keuls post hoc test for a significance level of $p \leq 0.05$. Results: After 10 sessions, increased flexibility was identified in the experimental groups $(p<0.01)$, but with no difference between them. Group $3 X$ significantly increased flexibility from the tenth day of the program (fifth session) and group $5 \mathrm{X}$ from the third day (third session). Conclusions: Stretching increases hamstring flexibility, regardless of the time between sessions ( 24 or 48 hours). Moreover, the interval between the sessions does not influence total flexibility gain. However, with five weekly stretching sessions, flexibility increases more rapidly than it does with three, suggesting that flexibility gain is session-dependent.
\end{abstract}

Keywords: proprioceptive neuromuscular facilitation, flexibility, stretching.

\section{INTRODUÇÃO}

Intervenções de alongamento visando aumentar a flexibilidade e a amplitude articular de movimento (AAM) têm sido preconizadas como importante componente em diferentes áreas relacionadas com a função motora. Seus efeitos benéficos estão demonstrados nas atividades funcionais ${ }^{(1,2)}$, na prevenção de lesões ${ }^{(3,4)}$, no treino postural e relaxamento muscular ${ }^{(5)}$, e no desempenho atlético ${ }^{(6)}$.

Dada sua importância, o treino de flexibilidade é alvo de numerosos estudos e publicações que abrangem a técnica de aplicação( ${ }^{(7-9)}$, os efeitos decorrentes ${ }^{(1-6)}$ e os mecanismos subjacentes à relação entre a técnica e o efeito ${ }^{(10-13)}$.

No que concerne à técnica, a busca da otimização está relacionada com a forma de alongamento e os parâmetros envolvidos em sua aplicação. Vários estudos compararam a melhor forma de alongamento, em sua maioria demonstrando vantagem no ganho de AAM para as técnicas que envolvem inibição ativa ou facilitação neuromuscular proprioceptiva (FNP), em relação às técnicas estática e balística, respectivamente ${ }^{(7-9)}$.

Quanto aos parâmetros, a duração da manobra de alongamento ${ }^{(10,11)}$ e o número de repetição da manobra por sessão ${ }^{(12,13)}$ foram as 
variáveis mais abordadas e discutidas em pesquisas anteriores. Entretanto, o intervalo de tempo entre as sessões de alongamento é um parâmetro ainda pouco estudado. Algumas pesquisas indicam que o ganho de flexibilidade pode ocorrer com diferentes intervalos entre as sessões, por exemplo, programas de alongamento diário, sete vezes por semana ${ }^{(14,15)}$, cinco ${ }^{(12,16)}$, três ${ }^{(7,8)}$ e até mesmo em programas com apenas duas sessões semanais ${ }^{(17,18)}$; porém, cumpre salientar que o efeito do intervalo entre as sessões não foi a variável estudada, sendo apenas um componente do protocolo experimental.

Imediatamente após uma sessão de alongamento ocorre aumento de flexibilidade, que parece ser perdido totalmente poucos minutos após o término de uma sessão ${ }^{(19-21)}$. Por isso, o intervalo de tempo após o estímulo de alongamento parece ser um fator que age contra o ganho de flexibilidade. Por outro lado, foi observado que um ganho residual persiste 24 horas depois de uma manobra de alongamento; contudo, o ganho residual obtido em uma única sessão não é estatisticamente significativo ${ }^{(22)}$. É possível que o aumento residual da flexibilidade seja decorrente da histerese inerente aos sistemas viscoelásticos ${ }^{(23)}$.

O propósito deste estudo foi verificar a efetividade de protocolos de alongamento com intervalos de repouso de 24 e 48 horas entre as sessões, ou seja, três e cinco vezes por semana, para aumentar a amplitude de extensão ativa do joelho (EAJ). Além disso, objetivou-se comparar a velocidade de ganho de flexibilidade relacionada com os dois protocolos. Maior entendimento desse assunto proporcionará condição para otimizar as sessões de treino de flexibilidade.

\section{METODOLOGIA}

\section{Sujeitos}

Participaram deste estudo 28 sujeitos do sexo feminino, jovens, saudáveis e sem disfunção para locomoção. Uma avaliação clínica inicial foi realizada no intuito de excluir os voluntários portadores de patologias osteomioarticulares, vasomotoras e com antecedentes de lesão articular ou deformidade no joelho direito (genuflexum, genu recurvatum, genu varum), além de deformidades na coluna vertebral ou lombalgia recente. Também foram excluídos os sujeitos que, na avaliação inicial, ultrapassaram a amplitude de $160^{\circ}$ de EAJ, com o quadril posicionado a $100^{\circ}$ de flexão. O ângulo de $180^{\circ}$ foi considerado como extensão completa do joelho. Os sujeitos não eram atletas e não participavam de qualquer programa de alongamento da musculatura posterior da coxa. Todos os sujeitos assinaram um termo de consentimento que informava sobre os possíveis riscos, reservando-Ihes o direito de desistência da pesquisa e assegurando-lhes o sigilo de sua identidade. Este estudo foi aprovado pelo Comitê de Ética e Pesquisa com seres humanos da instituição onde ocorreu a pesquisa.

A amostra foi distribuída aleatoriamente em três grupos, denominados grupo 0X ( $n=8)$, grupo $3 X(n=10)$ e grupo 5X $(n=10)$. O grupo 0X foi o controle e não participou dos programas de alongamento muscular. O grupo 3X foi submetido a sessões com intervalo de 48 horas (três vezes por semana) e o grupo 5X teve suas sessões intervaladas por 24 horas (cinco vezes por semana). Cada grupo experimental recebeu 10 sessões de alongamento muscular com FNP do tipo sustentar-relaxar nos isquiotibiais do membro direito e nenhum tipo de aquecimento prévio foi realizado. Não houve coleta de dados nos fins de semana e o horário das sessões era das 11:00 às 13:00 horas.

\section{PROCEDIMENTO EXPERIMENTAL}

\section{Instrumentos}

Foi utilizada uma prancha estabilizadora com uma câmera fotográfica acoplada, adaptada a partir do modelo desenvolvido por Brasileiro et al. ${ }^{(24)}$ e Gajdosik ${ }^{(14)}$ para medir a EAJ do membro direito. A prancha é composta por três segmentos de madeira e um eixo fixo, além de faixas fixadoras. Em uma extremidade foi adaptado um suporte de madeira para uma câmera digital. Esse suporte da câmera foi projetado para que a máquina ficasse rigorosamente em paralelo com o plano de movimento do membro avaliado. O sujeito era posicionado na prancha em decúbito dorsal e fixado no tórax, pelve, terço médio da coxa direita, e terços superior e inferior da coxa esquerda com o quadril direito flexionado $100^{\circ} \mathrm{e}$ apoiado em um segmento da prancha, de forma que este era posicionado para se adaptar ao comprimento da coxa do indivíduo. A fixação tinha o objetivo de evitar movimentos compensatórios durante a EAJ.

\section{Procedimento de medida}

No primeiro dia, um pesquisador localizou e demarcou quatro pontos de referência anatômica para o cálculo da EAJ no membro direito dos sujeitos. A localização e a marcação dos pontos foram feitas uma única vez com o sujeito posicionado em decúbito dorsal com o quadril e joelho em semiflexão. Foi utilizada uma tintura especial na pele dos participantes, que permaneceu por toda a pesquisa para garantir a mesma referência durante todo o período da coleta. Durante esta, marcadores adesivos foram usados sobre os pontos anatômicos demarcados pela tintura na coxa e perna do sujeito. Os referenciais anatômicos para fotometria da articulação do joelho foram determinados baseando-se em Gajdosik ${ }^{(14)}$, que desconsiderou pontos sobre as articulações por considerar que estas modificam seu eixo durante a movimentação do membro. Os marcadores adesivos foram posicionados, então, em quatro pontos anatômicos do membro avaliado: a $5 \mathrm{~cm}$ distais do trocânter maior, $5 \mathrm{~cm}$ proximais do epicôndilo femoral lateral, na cabeça da fíbula e no maléolo lateral. Foi realizado um estudo prévio, com oito sujeitos, onde as medidas obtidas com o instrumento mencionado (análises fotométricas) foram comparadas com um goniômetro universal acoplado a uma prancha de fixação. As medidas de EAJ apresentaram alto coeficiente de correlação $(r=0,99)$, demonstrando assim a confiabilidade dos dois instrumentos ${ }^{(25)}$.

Para a medição da EAJ o sujeito era fixado na prancha e realizava três extensões ativas máximas do joelho direito. No ponto final de cada extensão era registrada uma foto, que posteriormente era analisada com o auxílio do software AutoCAD ${ }^{\circledR} 2000$ (Autodesk, Inc., EUA, 2000). Duas retas eram traçadas, ambas unindo os marcadores anatômicos de cada segmento; uma ligando os marcadores fixados nos pontos sobre o fêmur e outra unindo os marcadores fixados sobre a fíbula. A média aritmética da dimensão angular formada entre as retas das três fotos correspondia à EAJ (figura 1). O grupo OX recebeu três medidas, uma no primeiro dia, outra 10 dias depois e a última, 22 depois da primeira. Os grupos experimentais, grupo $3 X$ e $5 X$, realizaram duas medidas por sessão, uma antes e outra depois do protocolo de alongamento. No total, foram analisadas 1.452 fotos durante a coleta dos dados.

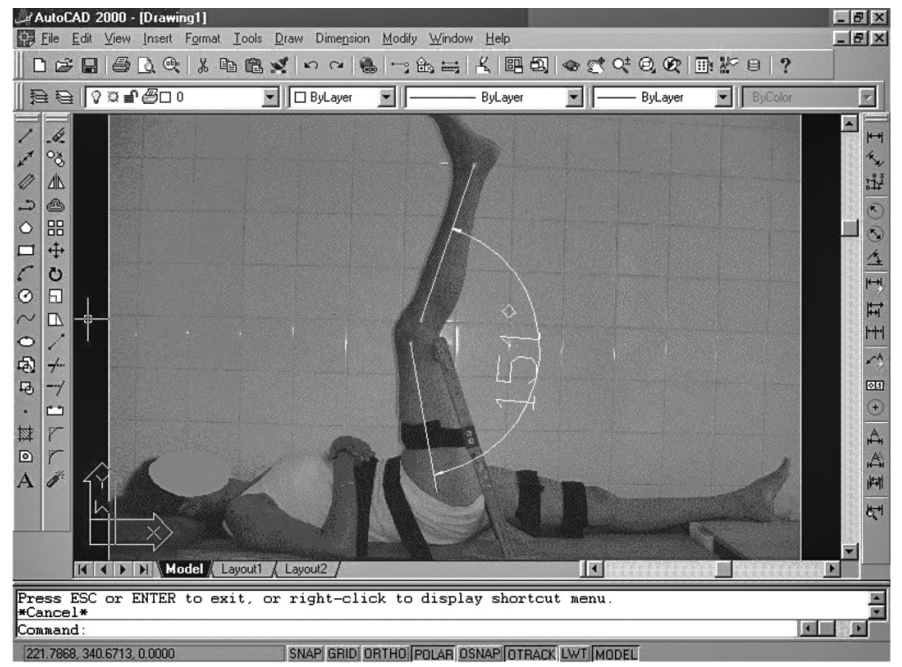

Figura 1. Medição da flexibilidade pelo cálculo da dimensão angular da extensão ativa do joelho direito. 


\section{Protocolo de alongamento}

As manobras de alongamento nos grupos experimentais foram realizadas por um único pesquisador. Os comandos verbais foram previamente padronizados e os sujeitos instruídos sobre a manobra antes do procedimento. O sujeito era colocado em decúbito dorsal e tinha sua coxa esquerda estendida e estabilizada pelo pesquisador com uma toalha (figura 2). O pesquisador flexionava o quadril direito do sujeito até a posição em que referisse início de desconforto (limiar de dor). Ao sinal do pesquisador era solicitada a realização de força máxima para estender o quadril, contraindo isometricamente os músculos extensores contra a resistência oferecida pelo pesquisador por cinco segundos. Ao final desses cinco segundos, o sujeito era instruído a relaxar a musculatura e logo em seguida tinha seu quadril flexionado passivamente até referir novo desconforto, sendo o membro mantido nesse ponto por 30 segundos. Cada sessão de alongamento foi composta por três manobras intercaladas por 10 segundos de repouso. Os sujeitos foram instruídos a relaxar a musculatura alongada durante a manobra para que não existisse resistência voluntária ao procedimento.

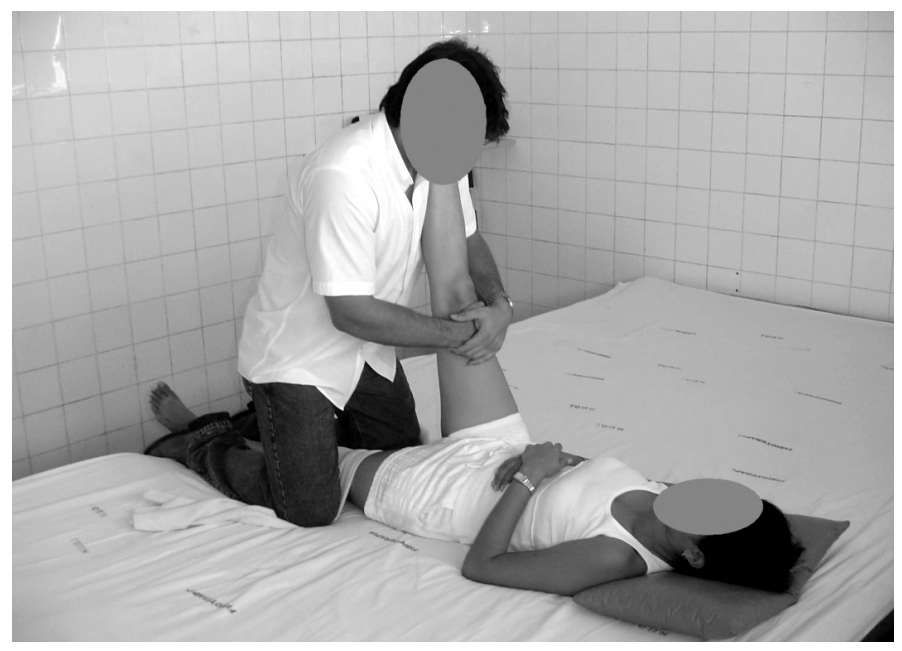

Figura 2. O sujeito era colocado em posição supina para receber o alongamento.

\section{ANÁLISE DOS DADOS}

Foi utilizada estatística descritiva para analisar os dados de idade, peso, altura, índice de massa corpórea, ganho total de flexibilidade, ganho imediato e ganho residual. Também foi utilizada análise de variância (ANOVA) unidirecional (one way) para testar a homogeneidade das medidas angulares iniciais (pré-teste) e também as características de idade, peso, altura e índice de massa corpórea.

Para avaliar o ganho total (pós-teste), imediato e residual, também foi utilizada análise de variância (ANOVA) unidirecional (one way) seguida do teste post hoc de Newman-Keuls conforme a necessidade.

Finalmente, os grupos experimentais foram submetidos a análise de variância (ANOVA) mista, de medidas parcialmente repetidas, com os dados de cada sessão para identificar o dia do primeiro ganho significativo de EAJ em relação à medida pré-teste. O grupo controle não foi incluído nessa análise específica por não possuir a mesma quantidade de medidas que os grupos experimentais. Em todos os testes o nível de significância adotado foi de $p \leq 0,05$. Os dados foram analisados com o software GBSTAT ${ }^{\circledR} 6.5$ (Dynamic Microsystems, Inc., EUA, 1997).

\section{RESULTADOS}

Os grupos se apresentaram homogêneos em relação à idade, peso, altura, índice de massa corpórea (IMC), como pode ser observado na tabela 1. A medida inicial de EAJ de cada grupo também foi estatisticamente semelhante $(p>0,05)$ (tabela 2).
Tabela 1. Características dos grupos quanto à média de idade, peso, altura e índice de massa corpórea (IMC).

\begin{tabular}{c|c|c|c|c|c}
\hline Grupos & N & Idade (ano) & $\begin{array}{c}\text { Peso } \\
\text { (quilograma) }\end{array}$ & $\begin{array}{c}\text { Altura } \\
\text { (metro) }\end{array}$ & IMC \\
\hline $0 X$ & 08 & $23,0 \pm 2,8$ & $52,4 \pm 5,8$ & $1,6 \pm 0,0$ & $19,9 \pm 1,9$ \\
\hline $3 X$ & 10 & $22,1 \pm 1,5$ & $54,3 \pm 4,7$ & $1,6 \pm 0,1$ & $22,0 \pm 1,4$ \\
\hline $5 X$ & 10 & $23,2 \pm 2,0$ & $51,5 \pm 6,0$ & $1,6 \pm 0,1$ & $21,0 \pm 1,3$ \\
\hline
\end{tabular}

Grupo 0X foi o controle, não alongou nenhum dia; Grupo 3X alongou três vezes por semana (48 horas de intervalo); Grupo 5X alongou cinco vezes por semana (24 horas de intervalo); \pm Desvio Padrão; Os dados foram coletados antes do programa de alongamento.

Tabela 2. Valores absolutos da EAJ (em graus) inicial, EAJ final e a variação da EAJ após as 10 sessões (ganho total) para os grupos experimentais e após 22 dias para o grupo controle $0 \mathrm{X}$.

\begin{tabular}{c|c|c|c|c}
\hline Grupos & N & EAJ inicial & EAJ final & Ganho tardio \\
\hline $0 X$ & 08 & $145,5 \pm 7,4$ & $146,7 \pm 7,4$ & $1,1 \pm 2,1$ \\
\hline $3 X$ & 10 & $144,7 \pm 7,2$ & $162,6^{\mathrm{a}} \pm 11,4$ & $17,9 \pm 10,9$ \\
\hline $5 X$ & 10 & $141,8 \pm 8,1$ & $156,3^{\mathrm{b}} \pm 9,4$ & $14,5 \pm 13,1$ \\
\hline
\end{tabular}

a diferença significativa em relação à EAJ inicial do grupo $5 X(p=0,001)$

b diferença significativa em relação à EAJ inicial do grupo $3 X(p=0,000)$ \pm Desvio Padrão

As principais variáveis dependentes analisadas neste estudo foram os ganhos imediato, residual e o ganho total. O ganho imediato é aumento de AAM imediatamente após cada sessão de alongamento. O ganho residual é o aumento de AAM entre duas sessões consecutivas de alongamento. $O$ ganho total é o aumento de AAM entre o primeiro dia (sem alongamento) e o último dia de intervenção após a realização de dez sessões.

O grupo controle não apresentou variação da EAJ entre a medida inicial e as medidas de 12 e 22 dias depois. Já o grupo 3X, no $22^{\circ}$ dia, e o grupo $5 X$, no $12^{\circ}$ dia, aumentaram significativamente em relação à medida inicial $(p<0,01)$. Contudo, comparando-se os valores de ganho total dos grupos 3X e 5X, não houve diferença estatisticamente significativa $(p>0,05)$.

Ao analisar o ganho imediato dos grupos, pode-se observar que o grupo $3 \mathrm{X}$ ganhava mais amplitude que o grupo 5X imediatamente depois do protocolo $(p=0,003)$. Todavia, parte desse ganho imediato se perdia entre uma sessão e outra, diminuindo em média 5,40 $\pm 1,4^{\circ}$ no grupo $3 X$ e 3,30 $\pm 1,4^{\circ}$ no grupo $5 X$. Esses dados representam diminuição de $81,2 \%$ e $74,6 \%$ do ganho imediato em cada grupo (figura 3).

O grupo $3 X$ manteve ganho residual médio de $1,3^{\circ} \pm 1,4^{\circ}$ e o grupo $5 X$, de $0,9^{\circ} \pm 1,6^{\circ}$ após cada sessão. Não ocorreu diferença significativa entre as médias de ganho residual $(p>0,05)$.

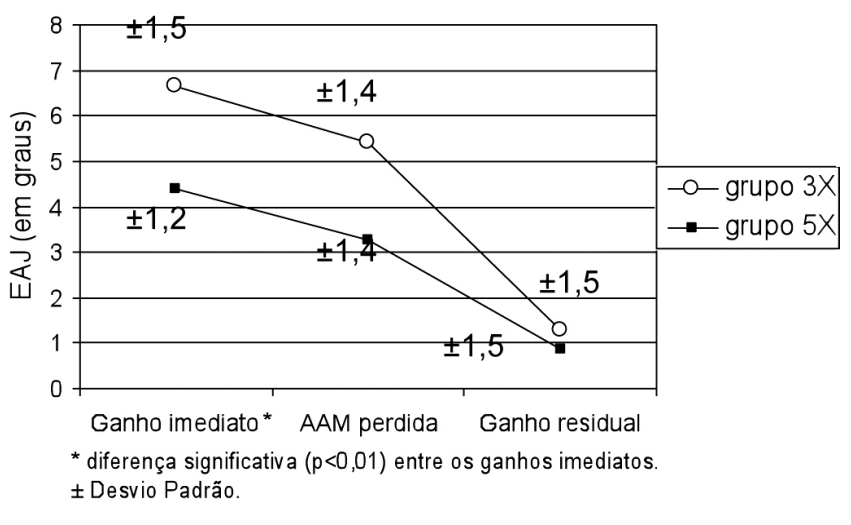

Figura 3. Perda média do ganho imediato 24 (grupo 5X) e 48 horas (grupos 3X) depois do alongamento. 
Ao se observar o conjunto de ganhos e perdas de flexibilidade ao longo do programa de alongamento, foi constatado que o somatório dos ganhos residuais, a cada sessão, é o fator que possibilita o ganho tardio de flexibilidade significativo.

A análise de variância ANOVA indicou que a somação diária do ganho residual do grupo $3 X$ resultou em aumento significativo ( $p=$ 0,037) da flexibilidade a partir do $10^{\circ}$ dia depois de iniciado o programa, que corresponde à quinta sessão de alongamento. Em relação ao grupo 5X, o ganho passou a ser significativo $(p=0,021)$ em três dias, que correspondeu ao terceiro dia de alongamento (figura 4).

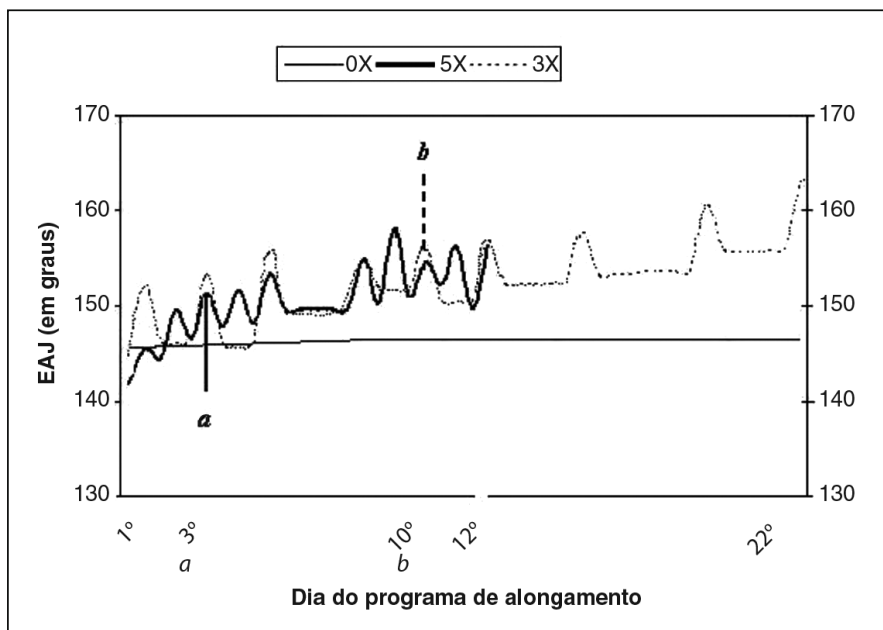

Figura 4. Curva da variação da EAJ dos grupos de alongamento e do grupo controle durante os 22 dias de experimento. O primeiro ponto de cada curva representa a primeira medida de EAJ sem alongamento, as cristas das ondas representam a EAJ imediatamente pós-alongamento e os vales a EAJ pré-alongamento do dia seguinte. (a) Primeiro dia de diferença significativa em relação à medida inicial ( $p=0,037)$ para o grupo 5X, equivalente a terceira sessão e terceiro dia do programa.

(b) primeiro dia de diferença significativa em relação à medida inicial $(p=0,021)$ para o grupo 3X, equivalente a quinta sessão e décimo dia do programa.

EAJ - Extensão Ativa do Joelho

\section{DISCUSSÃO}

Após um programa de treino de flexibilidade dos músculos isquiotibiais, consistindo de 10 sessões de alongamento e cuja distinção entre os grupos se dava apenas no intervalo de 24 ou 48 horas entre as sessões, os resultados indicaram que ambos os protocolos, dentro das condições deste estudo, são efetivos para o treino de flexibilidade e não determinam diferenças no ganho total de flexibilidade após essas 10 sessões do programa. A variável intervalo entre sessões não determinou diferenças significativas no ganho total de flexibilidade, foi diferente apenas na velocidade de ganho, indicando que o ganho total de flexibilidade dentro deste programa de alongamento foi sessão-dependente.

Embora a medida da eficácia da técnica de alongamento não seja objeto próprio deste estudo, e, portanto, não esteja o método direcionado para esse fim, os ganhos de EAJ dos grupos $3 X$ de $17,9^{\circ}$ e $5 X$ de 14,50, obtidos em 10 sessões, compreendidas em duas e pouco mais de três semanas, respectivamente, são aparentemente superiores a achados como o de Bandy et al.(12) e podem estar relacionados à junção de dois fatores de otimização: forma de alongamento, utilizando recurso de inibição autogênica descrito por Etnyre et al. ${ }^{(17)}$, entre outros pesquisadores ${ }^{(7-9)}$, e tempo de sustentação do alongamento ótimo, descrito por Bandy et al. ${ }^{(12)}$. Parece importante a confirmação de tal possibilidade por estudo dedicado a esse fim.

Após a sessão de alongamento ocorreram mudanças em curto prazo da flexibilidade, com aumento imediato da EAJ em ambos os grupos. Para Taylor et al.(23) e DeDeyne et al.(26), essas mudanças se devem às propriedades viscoelásticas da unidade musculotendínea. Contudo, outros autores sugerem que o ganho imediato de AAM com as manobras de alongamento deve-se mais às mudanças na tolerância do indivíduo ao alongamento que às alterações na elasticidade dos músculos ${ }^{(15,18,27)}$. Halbertsma et al. ${ }^{(15)}$ encontraram aumento significativo da AAM sem mudanças na curva de rigidez passiva do músculo, concluindo, por conseguinte, que o aumento da extensibilidade resultou de aumento na tolerância ao alongamento.

Durante o repouso, após o estímulo de alongamento, ocorre perda do efeito imediato. $O$ estudo de Spernoga et al. ${ }^{(20)}$ analisou a duração de tempo em que permanecia o ganho imediato após o alongamento dos isquiotibiais, com o uso da técnica de FNP sustentar-relaxar em apenas uma sessão, relatando assim que o ganho obtido com uma manobra de alongamento se perde totalmente seis minutos depois. Concluíram, então, que uma única manobra de alongamento não é suficiente para produzir deformação duradoura na unidade musculotendínea.

A perda do ganho imediato de flexibilidade dos isquiotibiais pode ser atribuída a alterações na região elástica do tecido (propriedades viscoelásticas) devido à tensão da manobra de alongamento(23) e também à influência das propriedades de tixotropia do músculo, promovida pelo repouso, favorecendo o encurtamento após um ganho de $\mathrm{AAM}^{(20)}$. Tixotropia é a propriedade de um tecido tornar-se mais líquido depois do movimento e retornar à rigidez, estado de gel, com o repouso. A tixotropia do músculo parece ser resultado de um aumento no número de ligações estáveis entre os filamentos de actina e miosina quando o músculo está em repouso(28,29).

Os achados da presente pesquisa também revelaram que a maior parte do efeito imediato do alongamento se perde em 24 horas (81,2\% de perda) ou em 48 horas (74,6\% de perda) depois. A análise do percentual de perda do ganho imediato indicou perda semelhante entre os dois grupos, revelando que a curva de perda do ganho imediato se mantém estável entre 24 e 48 horas de descanso. Esses resultados corroboram em parte os achados de Spernoga et al. ${ }^{(20)}$, Depino et al..(19) e Weijer et al.(21), porém, esses pesquisadores relataram perda total da AAM ganha pós-alongamento. Diferentemente, os resultados do presente estudo revelaram que, tanto 24 horas como 48 horas após cada sessão de alongamento, permanece um ganho residual não significativo. Os grupos $3 X$ e $5 X$ conservaram na sessão seguinte em média 17,2\% e 18,4\% do ganho imediato, respectivamente. É possível que o aumento residual da flexibilidade esteja relacionado com as propriedades viscoelásticas da unidade musculotendínea descritas por Taylor et al. ${ }^{(23)}$, sendo decorrente da diferença entre a energia absorvida pelas estruturas durante a tensão de alongamento e a energia dissipada após a retirada desta, determinando um aumento residual da flexibilidade por histerese inerente aos sistemas viscoelásticos. Porém, já que o aumento residual é relativamente pequeno, pode passar oculto em análises estatísticas que desconsiderem pequenos ganhos ${ }^{(19-21)}$.

Considerando que um intervalo de 48 horas (grupo 3X) não propicia encurtamento adaptativo maior que um intervalo de 24 horas (grupo 5X), depois do treino de alongamento muscular, pode-se observar que, para as condições deste estudo, a perda mais intensa do ganho imediato ocorreu nas primeiras 24 horas pós-alongamento. É possível que a perda ocorrida nos intervalos entre as sessões de 24 e 48 horas tenha se dado em sua maior parte nos primeiros minutos após o alongamento, a exemplo do identificado por Spernoga et al.(20), DePino et al. ${ }^{(19)}$ e Weijer et al. ${ }^{(21)}$. Assim, cessadas as modificações de curto prazo, a realização de uma nova sessão de alongamento pode promover a adição de um novo ganho residual e um aumento na velocidade de ganho de flexibilidade, como observado no protocolo diário em relação ao de três vezes por semana. Ou seja, o grupo 5X obteve ganho estatisticamente significativo de EAJ três dias após o início do programa, enquanto o grupo 3X, apenas dez dias depois. 
O resultado deste estudo pode ser importante para a prescrição de sessões de alongamento e sugere que em ambientes desportivos, ou em outros casos especiais em que o paciente ou o atleta necessite de reabilitação acelerada, deve-se prescrever o treino de flexibilidade com menores intervalos entre sessões. Uma vez que o treino de flexibilidade parece depender do número de sessões, não do intervalo entre elas, pode-se sugerir que, o quanto antes forem realizados os estímulos de alongamento, mais rapidamente se conseguirá o desejado aumento da flexibilidade muscular. Esses achados corroboram o estudo clínico de Malliaropoulos et al. ${ }^{(30)}$, que verificaram maior retorno da AAM pós-lesões dos isquiotibiais com protocolo de quatro sessões diárias em comparação com protocolo diário.

Entretanto, duas perguntas ainda carecem de resposta: qual o intervalo mínimo ótimo para treinamento de flexibilidade? E qual o intervalo máximo ótimo para treinamento de flexibilidade?

É importante destacar a relevância clínica do intervalo máximo, visto que, no que tange à ampla utilização do treinamento de flexibilidade em variadas situações clínicas, questões econômicas e de demanda concorrem para programações terapêuticas realizadas duas ou três vezes por semana.

Este estudo se resumiu aos intervalos de 24 e 48 horas entre as sessões de alongamento, porém, pesquisas futuras deverão analisar o efeito de intervalos menores que 24 horas, no intuito de acelerar ainda mais o treino de flexibilidade, a exemplo dos resultados de Malliaropoulos et al. ${ }^{(30)}$. Além disso, em situações em que se objetiva a manutenção da flexibilidade ou o fator tempo de tratamento não influi negativamente, poderá também analisar-se a efetividade de treinos de alongamento com intervalos maiores que 48 horas, por exemplo, treinos a serem realizados duas ou uma vez por semana.

Embora se perceba que um percentual da flexibilidade é mantido 48 horas após o alongamento, não foi analisada neste estudo a curva de perda da flexibilidade, tanto imediatamente após o alongamento quanto após o programa de 10 sessões. Willy et al. ${ }^{(16)}$ mostram que há perda considerável de AAM após interrupção de quatro semanas depois de treino de flexibilidade realizado durante seis semanas.

\section{REFERÊNCIAS BIBLIOGRÁFICAS}

1. Gajdosik RL, Vander Linden DW, McNair PJ, Williams AK, Riggin RJ. Effects of an eight-week stretching program on the passive-elastic properties and function of the calf muscles of older women. Clin Biomech. 2005;20(9):973-83.

2. Kerrigan DC, Xenopoulos-Oddsson A, Sullivan MJ, Lelas JJ, Riley PO. Effect of a hip flexor-stretching program on gait in the elderly. Arch Phys Med Rehabil. 2003;84(1):1-6.

3. Weldon SM, Hill RH. The efficacy of stretching for prevention of exercise-related injury: a systematic review of the literature. Man Ther. 2003;8(3):141-50. Review.

4. Herbert RD, Gabriel M. Effects of stretching before and after exercising on muscle soreness and risk of injury: systematic review. BMJ. 2002;325(7362):468. Review.

5. Lardner R. Stretching and flexibility: its importance in rehabilitation. J Bodywork Mov Ther. 2001;5:254-63.

6. Shrier I. Does stretching improve performance? A systematic and critical review of the literature. Clin J Sport Med. 2004;14(5):267-73. Review.

7. Sady SP, Wortman M, Blanke D. Flexibility training: ballistic, static or proprioceptive neuromuscular facilitation? Arch Phys Med Rehabil. 1982;63(6):261-3.

8. Davis DS, Ashby PE, McCale KL, McQuain JA, Wine, JM. The effectiveness of 3 stretching techniques on hamstring flexibility using consistent stretching parameters. J Strength Cond Res. 2005;19(1):27-32.

9. Funk DC, Swank AM, Mikla BM, Fagan TA, Farr BK. Impact of prior exercise on hamstring flexibility: a comparison of proprioceptive neuromuscular facilitation and static stretching. J Strength Cond Res. 2003;17(3):489-92.

10. Bandy WD, Iron JM. The effect of time on static stretch on the flexibility of the hamstring muscles. Phys Ther. 1994;74(9):845-50

11. Borms J, Van Roy P, Santens JP, Haentjens A. Optimal duration of static stretching exercises for improvement of coxo-femoral flexibility. J Sports Sci. 1987;5(1):39-47.

12. Bandy WD, Iron JM, Briggler $M$. The effect of time and frequency of static stretching on flexibility of the hamstring muscle. Phys Ther. 1997;77(10):1090-6.

13. Gama ZAS, Medeiros CAS, Dantas AVR, Souza TO. Influência da freqüência de alongamento utilizando facilitação neuromuscular proprioceptiva na flexibilidade dos músculos isquiotibiais. Rev Bras Med Esporte. 2007;13:33-38.

14. Gajdosik RL. Effects of static stretching on the maximal length and resistence to passive stretch of short hamstring muscles. J Orthop Sports Phys Ther. 1991;14(6):250-5.

15. Halbertsma JP, Göeken LN. Stretching exercises: effect on passive extensibility and stiffness in short hamstrings of healthy subjects. Arch Phys Med Rehabil. 1994;75(9):976-81.

16. Willy RW, Kyle BA, Moore SA, Chleboun GS. Effect of cessation and resumption of static hamstring muscle stretching on joint range of motion. J Orthop Sports Phys Ther. 2001;31(3):138-44.
Os resultados desta pesquisa se aplicam especificamente ao alongamento dos músculos isquiotibiais, pois, devido às particularidades de cada músculo, a resposta ao alongamento pode diferir. Fatores como a predominância do tipo de fibra muscular, a disposição das fibras, a estrutura do tendão e a biomecânica do tecido podem influir em reação diferente da unidade musculotendínea frente ao alongamento.

Finalmente, a amostra deste estudo era relativamente jovem, com média de idade de 22 anos e pequeno desvio padrão. Portanto, as conclusões deste estudo devem aplicar-se somente a grupos de idade similares e futuras pesquisas serão necessárias com sujeitos de outros grupos de idade, em particular com indivíduos idosos.

\section{CONCLUSÃO}

Protocolos de alongamento com intervalos de repouso de 24 ou 48 horas, ou seja, cinco ou três vezes por semana, foram efetivos para o aumento da flexibilidade dos isquiotibiais, usando a técnica de sustentar-relaxar por dez sessões. Além disso, o intervalo entre as sessões não influenciou na quantidade de ganho de EAJ. Entretanto, o grupo com intervalo menor entre as sessões aumentou a flexibilidade mais rapidamente, demonstrando que, quanto mais cedo se apliquem as sessões, mais depressa será o ganho de EAJ. Esses achados podem ter implicações clínicas no que concerne à prescrição de exercícios de alongamento, parecendo o ganho de flexibilidade ser dependente do número de sessões aplicadas e não do intervalo entre elas. Em futuros estudos, seria interessante avaliar a efetividade de protocolos com menores e maiores intervalos de descanso entre os exercícios, avaliar populações de diferentes faixas etárias, bem como outros grupos musculares e a repercussão das variáveis observadas sobre a curva de perda de flexibilidade após a interrupção do programa de alongamento.

Todos os autores declararam não haver qualquer potencial conflito de interesses referente a este artigo.

17. Etnyre BR, Lee EJ. Chronic and acute flexibility of men and women using three different stretching techniques. Res Q Exerc Sport. 1988;59:222-8.

18. Magnusson SP. Passive properties of human skeletal muscle during stretch maneuvers. A review. Scand J Med Sci Sports. 1998;8(2):65-77. Review.

19. Depino GM, Webright WG, Arnold BL. Duration of Maintained Hamstring Flexibility After Cessation of an Acute Static Stretching Protocol. J Athl Train. 2000;35(1):56-9.

20. Spernoga SG, Uhl TL, Arnold BL, Gansneder BM. Duration of Maintained Hamstring Flexibility After a One-Time, Modified Hold-Relax Stretching Protocol. J Athl Train. 2001;36(1):44-8.

21. De Weijer VC, Gorniak GC, Shamus E. The effect of static stretch and warm-up exercise on hamstring length over the course of 24 hours. J Orthop Sports Phys Ther. 2003;33(12):727-33.

22. Souza TO, Gama ZAS, Medeiros CAS, Dantas AVR. Comparação entre o ganho de flexibilidade imediata, incorporada e tardia dos músculos ísquios tibiais em mulheres saudáveis através da utilização de um protocolo de alongamento com facilitação neuromuscular proprioceptiva. In: Reunião anual da SBPC, 56., 2004, Cuiabá. Anais eletrônicos. São Paulo: SBPC/UFMT, 2005. Disponível em: <http://www.sbpcnet. org.br/livro/56ra>. Acesso em: 28 abr. 2008.

23. Taylor DC, Dalton JD, Jr Seaber AV, Garrett WE Jr. Viscoelastic properties of muscle-tendon units. The biomechanical effects of stretching. Am J Sports Med. 1990;18(3):300-9.

24. Brasileiro JS, Faria A, Queiroz LI. Influência do resfriamento e do aquecimento na flexibilidade dos músculos ísquios-tibiais. Rev Bras Fisioter. 2007;11:1-5.

25. Souza TO, Medeiros CAS, Gama ZAS, Dantas AVR, Sales ATN, Augusto DD. Análise da correlação entre dois instrumentos de medição da extensão do joelho. In: Reunião anual da SBPC, 56., 2004, Cuiabá. Anais eletrônicos. São Paulo: SBPC/UFMT, 2005. Disponivel em: <http://www.sbpcnet.org.br/livro/56ra>. Acesso em: 28 abr. 2008.

26. De Deyne PG. Application of passive stretch and its implications for muscle fibers. Phys Ther. 2001;81(2):819-27. Review.

27. Halbertsma JP, Mulder I, Goeken LN, Eisma WH. Repeated passive stretching: acute effect on the passive muscle moment and extensibility of short hamstrings. Arch Phys Med Rehabil. 1999;80(4):407-14

28. Walsh EG. Postural thixotropy: a significant factor in the stiffness of paralyzed limbs? Paraplegia. 1993;30:113-5.

29. Lakie M, Robson LG. Thixotropic changes in human muscle stiffness and the effects of fatigue. Q J Exp Physiol. 1988;73(4):487-500

30. Malliaropoulos N, Papalexandris S, Papalada A, Papacostas E. The role of stretching in rehabilitation of hamstring injuries: 80 athletes follow-up. Med Sci Sports Exerc. 2004;36(5):756-9. 